PROCEEDINGS OF THE

AMERICAN MATHEMATICAL SOCIETY

Volume 136, Number 1, January 2008, Pages 341-346

S 0002-9939(07)08650-9

Article electronically published on September 25, 2007

\title{
PROPERTY OF KELLEY FOR THE CARTESIAN PRODUCTS AND HYPERSPACES
}

\author{
JANUSZ J. CHARATONIK AND WŁODZIMIERZ J. CHARATONIK
}

(Communicated by Alexander N. Dranishnikov)

\begin{abstract}
A continuum $X$ having the property of Kelley is constructed such that neither $X \times[0,1]$, nor the hyperspace $C(X)$, nor small Whitney levels in $C(X)$ have the property of Kelley. This answers several questions asked in the literature.
\end{abstract}

A metric continuum $X$ is said to have the property of Kelley provided that for each point $x \in X$, for each subcontinuum $K$ of $X$ containing $x$ and for each sequence of points $x_{n}$ converging to $x$ there exists a sequence of subcontinua $K_{n}$ of $X$ containing $x_{n}$ and converging to the continuum $K$ (see e.g. 8, Definition 16.10, p. 538]).

The property, introduced by J. L. Kelley as property 3.2 in [7, p. 26], has been used there to study hyperspaces, in particular their contractibility (see e.g. Chapter 16 of [8] and Chapter V of [4], where references for further results in this area are given). Now the property, which has been recognized as an important tool in the investigation of various properties of continua, is interesting by its own right and has numerous applications to continuum theory. Many of them are not related to hyperspaces. In [9, Theorem 2.5, p. 293] Wardle has proved that homogeneous continua have the property of Kelley. In 3 the second-named author has generalized the property of Kelley to the non-metric case, and constructed an example showing that, unlike for metric continua, the homogeneity of non-metric ones does not imply the property of Kelley.

The property of Kelley for product spaces and for hyperspaces of a continuum was a subject of studies by a number of topologists for many years. Here we recall some of them. In [9, Corollary 4.6, p. 297] it is proved that if a product of two continua has the property of Kelley, then each factor space has the property of Kelley. The converse is not true: the union $X$ of two opposite spirals in the plane approaching the unit circle has the property of Kelley, while $X \times X$ does not; see [9. Example 4.7, p. 297]. Presenting this example in his book ([8, Example 16.35, p. 558]), Nadler writes, "It seems quite surprising that the Cartesian product of two continua, each having the property of Kelley, may fail to have the property of Kelley." Further investigations in the area lead to formulate some basic questions: If a continuum $X$ has the property of Kelley and a continuum $Y$ is locally connected, does $X \times Y$ have the property of Kelley (see [5, Question 1, p. 173])? Or even, Does

Received by the editors July 9, 2004, and, in revised form, January 15, 2006.

2000 Mathematics Subject Classification. Primary 54B10, 54B20, 54F15.

Key words and phrases. Cartesian product, continuum, hyperspace, property of Kelley. 
$X \times[0,1]$ have the property of Kelley (see [6. Problem 3.4, p. 1148] and compare 4, Question 50.2, p. 277])? Both of these questions are answered in the negative by our Theorem 1 .

The notion of the property of Kelley is important in hyperspace theory. In 8. Questions 16.37, p. 558] Nadler asks about relations between the following assertions: (a) the continuum $X$ has the property of Kelley, (b) the hyperspace $2^{X}$ has the property of Kelley, (c) the hyperspace $C(X)$ has the property of Kelley. In 22 the second-named author showed that there is a curve $X$ such that $X$ and $C(X)$ have the property of Kelley while $2^{X}$ does not (in fact, $X$ is the circle with two spirals mentioned above). Here we show that the implication from (a) to (c) does not hold in general.

In [9, p. 295] Wardle asks whether the property of Kelley is a Whitney property, that is, if $X$ has the property of Kelley, then for each Whitney map $\mu: C(X) \rightarrow$ $[0, \infty)$ each Whitney level $\mu^{-1}(t)$ also has the property. Recalling the question in 4, Question 50.1, p. 277] Illanes and Nadler comment: "This problem has turned out to be very difficult." Trying to solve the question, Kato has proved in [6, Corollary 3.3 , p. 1147] that in order to obtain an affirmative answer to the question (i.e., to show that the property of Kelley is a Whitney property) it is enough to show that if $X$ has the property of Kelley, then $X \times[0,1]$ has the property. Thus problems concerning the property of Kelley for hyperspaces are tied with ones for product spaces. It is shown in Theorem 1 below that the property of Kelley for $X$ does not imply the property for $X \times[0,1]$ or for Whitney levels.

Let us recall the needed definitions and notation.

All considered spaces are assumed to be metric. We denote by $\mathbb{N}$ the set of all positive integers, and by $\mathbb{R}$ the space of reals. A continuum means a compact connected space, and a mapping means a continuous function. Given a subset $A$ of a space $X$ and a positive real number $\varepsilon$, we denote by $N(A, \varepsilon)$ an $\varepsilon$-neighborhood of $A$ in $X$.

Given a continuum $X$, we let $2^{X}$ denote the hyperspace of all non-empty closed subsets of $X$ equipped with the Hausdorff metric $H$ (see e.g. 8, (0.1), p. 1 and (0.12), p. 10]). Further, we denote by $C(X)$ the hyperspace of all subcontinua of $X$, i.e., of all connected elements of $2^{X}$.

A Whitney map for $C(X)$ is a mapping $\mu: C(X) \rightarrow[0, \infty)$ such that:

(0.1) $\mu(A)<\mu(B)$ for every two $A, B \in C(X)$ such that $A \subset B$ and $A \neq B$;

(0.2) $\mu(A)=0$ if and only if $A$ is a singleton.

For the concept and existence of a Whitney map see [4, Section 13, pp. 105-110]. For each $t \in[0, \mu(X)]$ the preimage $\mu^{-1}(t)$ is called a Whitney level. It is known that each Whitney level is a continuum; see [4, p. 159].

The reader is referred to monographs [4 and 8 for definitions and basic properties of other notions used in the paper.

The main result of this paper is the following theorem.

Theorem 1. There exists a continuum $X$ having the following properties.

(11) $X$ has the property of Kelley;

(12) $X \times[0,1]$ does not have the property of Kelley;

(113) the hyperspace $C(X)$ does not have the property of Kelley;

(114) for each Whitney map $\mu: C(X) \rightarrow[0, \infty)$ there exists a number $s>0$ such that for each $t \in(0, s)$ the Whitney level $\mu^{-1}(t)$ does not have the property of Kelley. 


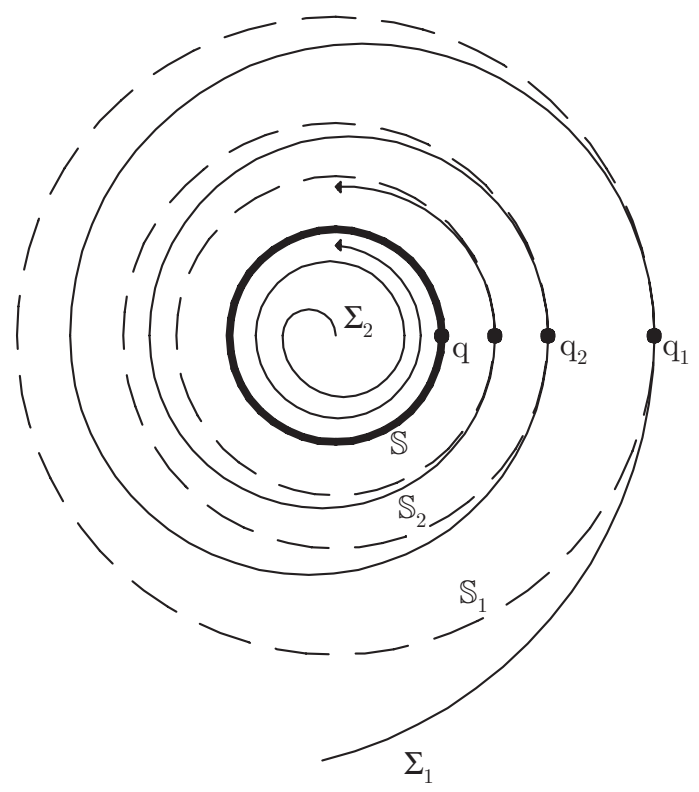

FiguRE 1.

Proof. In the polar coordinates $(r, \varphi)$ in the plane consider the circles

$$
\begin{aligned}
S & =\{(r, \varphi): r=1\} \quad \text { (the thick circle), } \\
S_{n} & =\left\{(r, \varphi): r=1+\frac{1}{2 \pi n}\right\} \text { for } n \in \mathbb{N} \quad \text { (the dashed circles) }
\end{aligned}
$$

and two spirals

$$
\begin{aligned}
& \Sigma_{1}=\left\{(r, \varphi): r=1+\frac{1}{\varphi} \text { and } \varphi \in[1, \infty)\right\}, \\
& \Sigma_{2}=\left\{(r, \varphi): r=1-\frac{1}{\varphi} \text { and } \varphi \in[1, \infty)\right\}
\end{aligned}
$$

pictured in Figure 1 as solid lines.

Thus $\Sigma_{1}$ and $\Sigma_{2}$ go in the same direction and approach the unit circle $S$ from outside $\left(\Sigma_{1}\right)$ and from inside $\left(\Sigma_{2}\right)$.

The constructed continuum $X$ is defined as

$$
X=\Sigma_{1} \cup \Sigma_{2} \cup S \cup \bigcup\left\{S_{n}: n \in \mathbb{N}\right\} .
$$

For each $n \in \mathbb{N}$ define $q_{n}=\left(1+\frac{1}{2 \pi n}, 0\right)$ and observe that $S_{n} \cap \Sigma_{1}=\left\{q_{n}\right\}$ and $\lim q_{n}=q=(1,0)$.

Further, denote by $p: X \rightarrow S$ the central projection defined by $p((r, \varphi))=(1, \varphi)$.

1 ) To show that $X$ has the property of Kelley consider a point $x \in X$, a sequence of points $x_{n} \in X$ tending to $x$ and a continuum $K \subset X$ containing the point $x$. We have to show that there are continua $K_{n}$ such that $x_{n} \in K_{n}$ for each $n \in \mathbb{N}$ and $K=\operatorname{Lim} K_{n}$.

If $x \in X \backslash S$, then $X$ is locally connected at $x$, and we may take as $K_{n}$ the union of $K$ and the shortest arc in $X$ joining $x_{n}$ and $x$. If $x \in S$ we need to consider two cases. 
CASE 1. $K \subsetneq S$. For each $n \in \mathbb{N}$, let $P_{n}$ be an irreducible arc in $S$ from $p\left(x_{n}\right)$ to $K$. Note that $\lim \operatorname{diam}\left(P_{n}\right)=0$. Then it is enough to define $K_{n}$ as the component of $p^{-1}\left(K \cup P_{n}\right)$ containing $x_{n}$.

CASE 2. $S \subset K$. Then for each $n \in \mathbb{N}$ there is a spiral $\Sigma_{n}^{\prime}$ having $x_{n}$ as its end point and approaching $S$. Indeed, if $x_{n} \in \Sigma_{1}$, then $\Sigma_{n}^{\prime}$ is a subspiral of $\Sigma_{1}$; if $x_{n} \in \Sigma_{2}$, then $\Sigma_{n}^{\prime}$ is a subspiral of $\Sigma_{2}$; and if $x_{n} \in S_{k}$ for some $k \in \mathbb{N}$, then $\Sigma_{n}^{\prime}$ is the union of an arc joining $x_{n}$ to $\Sigma_{1}$ contained in $S_{k}$ and a subspiral of $\Sigma_{1}$. Finally put $K_{n}=K \cup \Sigma_{n}^{\prime}$. Since the spirals $\Sigma_{n}^{\prime}$ tend to $S$, we have $K=\operatorname{Lim} K_{n}$. So, we have shown that $X$ has the property of Kelley.

2) To show that $X \times[0,1]$ does not have the property of Kelley, define (in the cylindrical coordinates $(r, \varphi, z)$ in the 3-space) an arc

$$
A=\{(1,2 \pi z, z): z \in[0,1]\} \subset S \times[0,1] .
$$

Further, for each $n \in \mathbb{N}$, define

$$
A_{n}=\left\{(r, \varphi, z): r=1+\frac{1}{\varphi+2 \pi n}, \varphi=2 \pi z \text {, and } z \in[0,1]\right\} .
$$

Thus $A_{n}$ is an arc from $\left(q_{n}, 0\right)$ to $\left(q_{n+1}, 1\right)$ lying in $\Sigma_{1} \times[0,1]$.

Similarly, for each $n \in \mathbb{N}$, define

$$
B_{n}=\left\{(r, \varphi, z): r=1+\frac{1}{2 \pi n}, \varphi=2 \pi z \text { and } z \in[0,1]\right\} .
$$

Thus $B_{n}$ is an arc with end points $\left(q_{n}, 0\right)$ to $\left(q_{n}, 1\right)$ lying in $S_{n} \times[0,1]$.

Note that

$$
A_{n} \cap B_{n}=\left\{\left(q_{n}, 0\right)\right\} \text { and } A_{n} \cap B_{n+1}=\left\{\left(q_{n+1}, 1\right)\right\} .
$$

Finally put

$$
\begin{gathered}
K=A \cup \bigcup\left\{A_{n} \cup B_{n}: n \in \mathbb{N}\right\}, \quad x=(1,0,0), \\
x_{n}=\left(1-\frac{1}{2 \pi n}, 0,0\right) \text { for each } n \in \mathbb{N},
\end{gathered}
$$

and observe that $x \in K, x_{n} \in \Sigma_{2} \times[0,1]$ and $x=\lim x_{n}$.

We will show that there are no continua $K_{n} \subset X \times[0,1]$ converging to $K$ and containing the points $x_{n}$. To this aim suppose on the contrary that there are such continua and observe that $(p \times \mathrm{id})(K)=A$. Then for all but finitely many $n$ we must have $K_{n} \subset(p \times \mathrm{id})^{-1}(N(A, \varepsilon))$ for arbitrary $\varepsilon>0$. Note that, however, for $\varepsilon<1$ the component of $(p \times \mathrm{id})^{-1}(N(A, \varepsilon))$ that contains $x_{n}$ is a subset of $\Sigma_{2} \times[0,1]$, so $K_{n} \subset \Sigma_{2} \times[0,1]$. Hence, if $d$ denotes the metric in $X \times[0,1]$ and $H$ denotes the Hausdorff metric, we have

$$
H\left(K, K_{n}\right) \geq d\left((q, 0),\left(q_{1}, 0\right)\right)=\frac{1}{2 \pi} \quad \text { for each } n \in \mathbb{N},
$$

whence it follows that $K$ is not the limit of continua $K_{n}$. This completes the proof that $X \times[0,1]$ does not have the property of Kelley.

3) To show that the hyperspace $C(X)$ does not have the property of Kelley, accept the following notation, where $n \in \mathbb{N}$ :

$$
\begin{aligned}
D_{\alpha} & =\{(1, \varphi): \varphi \in[\alpha, \alpha+\pi]\} \subset S, \text { where } \alpha \in \mathbb{R}, \\
\mathcal{A} & =\left\{D_{\alpha}: \alpha \in[0, \pi]\right\} \subset C(S) .
\end{aligned}
$$

Thus $D_{\alpha}$ is an arc in the circle $S$ and $\mathcal{A}$ is an arc in the hyperspace $C(S)$. Define

$$
\mathcal{K}=\left\{Q \in C\left(X \backslash \Sigma_{2}\right): p(Q) \in \mathcal{A}\right\}
$$

and observe that $\mathcal{K}$ is the union of a ray in $C(X)$ approximating the arc $\mathcal{A}$ and of $\mathcal{A}$. Take $D_{0} \in \mathcal{K}$. Let $D_{n}^{\prime}$ be a sequence of arcs in the inner spiral $\Sigma_{2}$ tending to $D_{0}$. 
Then arguing as previously one can show that there is no sequence of subcontinua $\mathcal{K}_{n}$ of $C(X)$ such that $D_{n}^{\prime} \in \mathcal{K}_{n}$ and having $\mathcal{K}$ as its limit. Thus $C(X)$ does not have the property of Kelley.

4) Let $\mu: C(X) \rightarrow[0, \infty)$ be an arbitrary Whitney map, and define $s=\mu(S)$. To show (1.4) we may modify the above proof of (1.3) in such a way that for each number $t$ with $0<t<s$ the continua $D_{\alpha}$ are arcs in the Whitney level $\mu^{-1}(t)$; similarly the $\operatorname{arc} \mathcal{A}$ and the ray $\mathcal{K}$ can be taken as subsets of $\mu^{-1}(t)$. Moreover, we may consider $\operatorname{arcs} D_{n}^{\prime}$ in $C\left(\Sigma_{2}\right)$ such that $\mu\left(D_{n}^{\prime}\right)=t$ and $\operatorname{Lim} D_{n}^{\prime}=D_{0}$. Then there are no continua $\mathcal{K}_{n} \subset \mu^{-1}(t)$ containing $D_{n}^{\prime}$ such that $\mathcal{K}=\operatorname{Lim} \mathcal{K}_{n}$.

This finishes the proof.

Theorem 1 implies the following corollaries.

Corollary 2. If a continuum $X$ has the property of Kelley, then the product $X \times$ $[0,1]$ need not have the property of Kelley.

Corollary 2 answers in the negative a question of Kato in [6, Problem 3.4, p. 1148] (compare also [4, Question 50.2, p. 277]).

Corollary 3. If a continuum $X$ has the property of Kelley, then the hyperspace $C(X)$ need not have the property of Kelley.

Corollary 3 answers in the negative a question of Nadler in [8, Questions 16.37, p. 558] (repeated in [4, Questions 78.27, p. 405]).

A topological property $\mathcal{P}$ is said to be a small weak Whitney property provided that for each continuum $X$ there are a Whitney map $\mu: C(X) \rightarrow[0, \infty)$ and a number $s \in(0, \mu(X))$ such that if $X$ has the property $\mathcal{P}$, then for each $t \in[0, s)$ the Whitney level $\mu^{-1}(t)$ has the property $\mathcal{P}$, [1].

Corollary 4. The property of Kelley is not a small weak Whitney property, and thus it is not a Whitney property.

Corollary 4 answers in the negative the question of Wardle in [9, p. 295].

We close the paper with some open questions related to the subject.

In [8, Questions 16.37, p. 558] Nadler asks several questions concerning implications between the property of Kelley for a continuum $X$ and the hyperspaces $2^{X}$ and $C(X)$. All these questions have been already answered except one.

Question 5 (Nadler). Let $X$ be a continuum. If $2^{X}$ has the property of Kelley, then does $C(X)$ have the property?

In a conversation with the second-named author, J. R. Prajs asked the following two questions.

Questions 6 (Prajs). Let $X$ be a continuum. a) If $X \times[0,1]$ has the property of Kelley, then does $C(X)$ have the property? b) If $C(X)$ has the property of Kelley, then does $X \times[0,1]$ have the property?

Question 7. Let $X$ be a continuum and let $\mu: C(X) \rightarrow[0, \infty)$ be a Whitney map. If, for each $t>0$, the Whitney level $\mu^{-1}(t)$ has the property of Kelley, then does $C(X)$ have the property?

A topological property $\mathcal{P}$ is said to be a sequential strong Whitney reversible property provided that whenever $X$ is a continuum such that there is a Whitney map $\mu$ for $C(X)$ and a sequence $\left\{t_{n}: n \in \mathbb{N}\right\}$ such that $\lim t_{n}=0$ and $\mu^{-1}\left(t_{n}\right)$ has 
property $\mathcal{P}$ for each $n$, then $X$ has property $\mathcal{P}$, 4, Definition 27.1 (d), p. 232 and 233].

It is known that the property of Kelley is a sequential strong Whitney reversible property; see [4, Theorem 50.4, p. 277]. The next question is related to this result.

Question 8. Let $X$ be a continuum. If there are a Whitney map $\mu$ for $C(X)$ and a sequence $\left\{t_{n}: n \in \mathbb{N}\right\}$ such that $\lim t_{n}=0$ such that $\mu^{-1}\left(t_{n}\right)$ has the property of Kelley for each $n$, then does $C(X)$ have the property?

\section{ACKNOWLEDGMENT}

The first-named author recognizes that the whole idea of the construction of the continuum $X$ in Theorem 1 is due to the second-named author.

\section{REFERENCES}

[1] J. J. Charatonik and W. J. Charatonik, Weak and small Whitney properties, Topology Proc. 28 (2004), 69-79. MR2105448 (2005g:54018)

[2] W. J. Charatonik, Hyperspaces and the property of Kelley, Bull. Polish Acad. Sci. Math. 30 (1982), 457-459. MR0703573 (85c:54052)

[3] W. J. Charatonik, A homogeneous continuum without the property of Kelley, Topology Appl. 96 (1999), 209-216. MR1709689 (2000i:54044)

[4] A. Illanes and S. B. Nadler, Jr., Hyperspaces, M. Dekker, New York and Basel, 1999. MR1670250 (99m:54006)

[5] H. Kato, On the property of Kelley in the hyperspace and Whitney continua, Topology Appl. 30 (1988), 165-174. MR0967753 (90b:54006)

[6] H. Kato, A note on continuous mappings and the property of J. L. Kelley, Proc. Amer. Math. Soc. 112 (1991), 1143-1148. MR.1073527 (91j:54013)

[7] J. L. Kelley, Hyperspaces of a continuum, Trans. Amer. Math. Soc. 52 (1942), 22-36. MR0006505 (3:315b)

[8] S. B. Nadler, Jr., Hyperspaces of sets, M. Dekker, New York and Basel, 1978. MR0500811 $(58: 18330)$

[9] R. W. Wardle, On a property of J. L. Kelley, Houston J. Math. 3 (1977), 291-299. MR0458379(56:16582)

Mathematical Institute, University of WrocŁaw, WrocŁaw, Poland; and Instituto de Matemàticas, UNAM, Cuidad Universitaria, Mexico

Department of Mathematics and Statistics, University of Missouri-Rolla, Rolla, Missouri 65409-0020

E-mail address: wjcharat@umr.edu 